\title{
Mössbauer effect study of tight spin coupling in oxidized chloro-5,10,15,20-tetra(mesityl)porphyrinatoiron(III)
}

\author{
Brian Boso and George Lang \\ Department of Physics, Pennsylvania State University, University Park, Pennsylvania 16802
}

Thomas J. McMurry and John T. Groves

Department of Chemistry, University of Michigan, Ann Arbor, Michigan 48109

(Received 2 December 1982; accepted 18 March 1983)

\begin{abstract}
Mössbauer spectra of a polycrystalline form of oxidized chloro-5,10,15,20-tetra/mesityl/porphyrinatoiron(III) $[\mathrm{Fe}(\mathrm{TMP}) \mathrm{Cl}]$, compound $\mathrm{A}$, were recorded over a range of temperatures $(4.2-195 \mathrm{~K})$ and magnetic fields $(0-6 \mathrm{~T})$. These spectra of compound A exhibit magnetic features which are markedly different from those of the analogous protein complexes, horse radish peroxidase compound I (HRP-I) and compound ES of cytochrome $c$ peroxidase, even though chemical evidence and optical spectroscopy indicate that compound $\mathrm{A}$ is similar to the others in comprising a $\mathrm{Fe}(\mathrm{IV})$ complex within a porphyrin cation radical. We interpret the data by employing a spin Hamiltonian model in which the central Fe(IV) complex, with $S=1$, is tightly coupled to a $S=1 / 2$ system of the oxidized porphyrin to yield a net $S=3 / 2$ system as suggested by the susceptibility measurements. The theoretical treatment yields information on the $d$-electron energies which is similar to that more directly available in the peroxidase spectra. The strength of the axial crystal field is found to increase progressively in the series HRP-I, ES, compound A, while the spin coupling in A is the strongest of the three by several orders of magnitude. The good fits to experimental data confirm the efficacy of the theoretical treatment which may be applicable to a variety of other coupled systems.
\end{abstract}

\section{INTRODUCTION}

The catalytic cycles of a number of heme proteins involve high valence states of iron. ${ }^{1}$ In some cases these are accompanied by a second paramagnetic site produced by the removal of an electron from the adjacent structure. The observation of intramolecular spin coupling in some of these enhances their technical interest. At the same time the growing number of heme and nonheme proteins which exhibit spin coupling makes worthwhile the development and testing of methods to recognize these interactions and to interpret the resulting Mössbauer spectra.

The two-electron oxidation of cytochrome $c$ peroxidase to compound ES by hydrogen peroxide results in the conversion of $\mathrm{Fe}$ (III) to $\mathrm{Fe}(\mathrm{IV})$, while the second electron is apparently removed from a site remote from the iron. ${ }^{2}$ The extremely weak coupling between the iron and the free radical has led to the suggestion that the latter is located on the protein, possibly methionine 171, rather than on the porphyrin. ${ }^{3}$ By contrast, spin coupling of a few $\mathrm{cm}^{-1}$ has been detected by EPR, Mössbauer, and ENDOR measurements ${ }^{4,5}$ in horseradish peroxidase compound I (HRP-I). Further, the NMR hyperfine shifts observed for this species and for the HRP-I reconstituted with deuterohemin were consistent with $\pi$ spin density on the porphyrin. ${ }^{6}$ Thus a porphyrin $\pi$-cation radical, as originally inferred from the visible spectrum, ${ }^{7}$ is the most likely structure of HRP-I. Several lines of evidence have suggested that the active oxygen species of cytochrome P-450 is also an oxyferryl species related to HRP-I although no such intermediate has been detected spectroscopically. ${ }^{8,9}$

It has recently become possible to synthesize simple oxidized iron-porphyrin complexes. The low temperdture oxygenation of synthetic iron(II) porphyrins has led to the generation of iron(IV) porphyrin complexes of the same oxidation state as HRP compound II. ${ }^{10}$ Mössbauer measurements on these species indicate a central iron atom very similar to that of ES. ${ }^{11}$ The first synthetic porphyrin complex with spectral properties similar to those of HRP-I has recently been reported. ${ }^{12}$ Oxidation of the sterically protected chloro-5, 10, 15, 20-tetra(mesityl) porphyrinatoiron(III) $[\mathrm{Fe}(\mathrm{TMP}) \mathrm{Cl} \mid$ with $m$ chloroperoxybenzoic acid produced a green species, compound $\mathrm{A}$, with a visible spectrum characteristic of a prophyrin cation radical. Solution magnetic susceptibility measurements gave $\mu_{\text {etf }}=4.2 \mu_{B}$, very near to that predicted for a free spin $3 / 2$ system. The Mössbauer spectra of compound A show an isomer shift and quadrupole splitting similar to the iron(IV) of compound ES. Further, compound $A$ was observed to react with olefins at low temperatures to yield epoxides and to regenerate the starting $\mathrm{Fe}(\mathrm{TMP}) \mathrm{Cl}$.

We present here further Mössbauer studies on compound $\mathrm{A}$ in frozen toluene solution and show that the paramagnetic features of the spectra differ markedly from those of ES and HRP-I. Detailed interpretation confirms the theoretical method, reveais the presence of a spin $3 / 2$ system, indicates that the spin coupling is the principal cause of the Mössbauer spectral differences, and shows that the central ferryl complex is indeed very similar to those of the proteins. 


\section{EXPERIMENTAL}

\section{Sample preparation}

$5,10,15,20$-Tetra(mesityl)porphyrin $\left(\mathrm{TMPH}_{2}\right)$ was prepared according to the method of Badger ${ }^{13}$ with improvements we have described elsewhere. ${ }^{14} \mathrm{TMPH}_{2}$ was metallated with ${ }^{57} \mathrm{FeBr}_{2}(80 \%$ enriched) in THF at room temperature. ${ }^{15}$ The crude metalloporphyrin was treated with sodium hydroxide, and eluted with methylene chloride from Merck basic alumina (activity IV). The resulting iron(III) hydroxide $[\mathrm{Fe}(\mathrm{TMP}) \mathrm{OH}]^{16,12}$ was converted to chloro-5, 10, 15, 20-tetra(mesityl) porphyrinatoiron(III) [Fe(TMP)Cl] with concentrated hydrochloric acid. The purity of the resulting $\mathrm{Fe}(\mathrm{TMP}) \mathrm{Cl}$ was confirmed by noting the presence of a single pair of sharp resonances in the room temperature ${ }^{1} \mathrm{H}$-NMR spectrum at $\delta 14.3$ and 16.0 due to the metahydrogens and a broad symmetrical resonance at $\delta 80.6$ due to the $\beta$-pyrrole hydrogens.

The oxidation of $\mathrm{Fe}$ (TMP)Cl was carried out in toluene$d_{8}$ at $-90^{\circ} \mathrm{C}$ by the addition of $2.75 \mathrm{eq}$ of $\mathrm{m}$-chloroperoxybenzoic acid in methanol- $d_{4}$. The ${ }^{1} \mathrm{H}-\mathrm{NMR}$ spectra of the green solutions produced in this manner showed the characteristic resonances we have ascribed to the iron(IV) porphyrin cation radical species, compound $\mathrm{A},{ }^{12}$ and no resonances due to the starting iron(III) chloride. This solution was cooled to $-98^{\circ} \mathrm{C}$, transferred into an $1 / 8$ inch polyethylene Mössbauer cell, and immediately frozen at $77 \mathrm{~K}$.

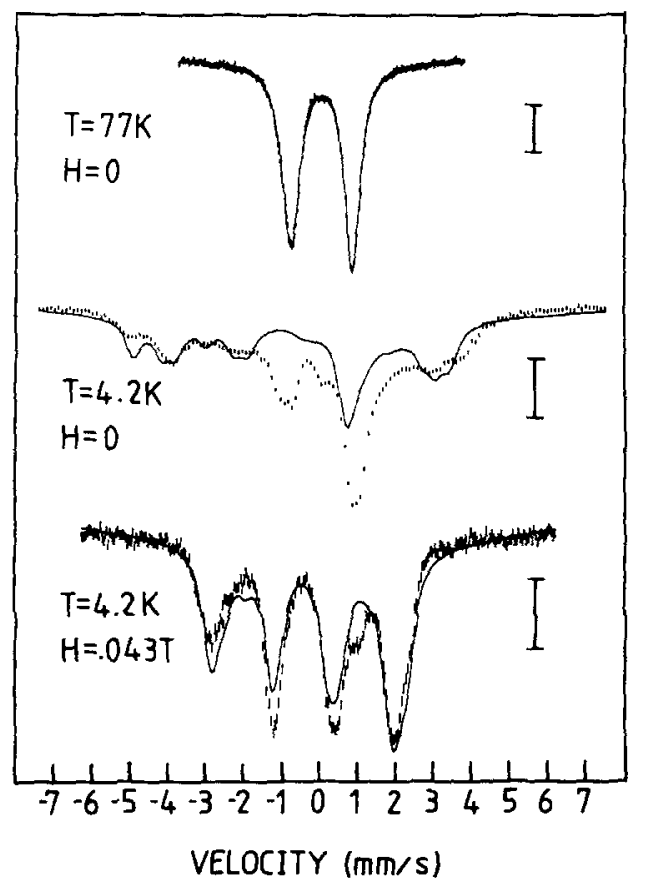

FIG. 1. Mössbauer spectra of compound $A$ in zero applied magnetic field and a small transverse magnetic field. The $77 \mathrm{~K}$ spectrum is fit with two Lorentzians, while the calculation of the $4.2 \mathrm{~K}$ spectra is based on the $S=3 / 2$ spin Hamiltonian of Eqs. (1) and (2). The bars at the right indicate $1 \%$ resonant absorption.

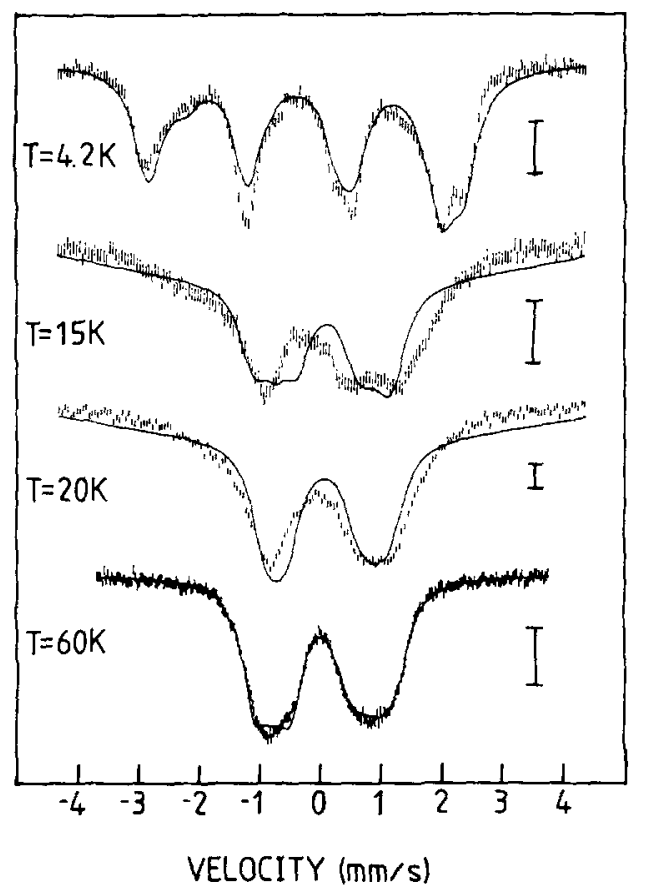

FIG. 2. Mössbauer spectra of compound A in a transverse magnetic field of $4 \mathrm{~T}$ at temperatures indicated. The solid lines are calculations based on the $S=3 / 2$ spin Hamiltonian of Eqs. (1) and (2). The bars at the right indicate $1 \%$ resonant absorption.

\section{Mössbauer measurements}

Mössbauer spectra were recorded in horizontal transmission geometry using a constant acceleration spectrometer operated in synchronization with a 256 channel analyzer in the time scale mode. The source of gamma radiation was approximately $25 \mathrm{mCi}$ of ${ }^{57} \mathrm{Co}$ diffused in rhodium and was kept at room temperature for all experiments. The velocity scale of the spectrometer was calibrated with an iron foil at room temperature and the centroid of the iron spectrum is taken as the zero of velocity for all data reported herein. Typical calibration linewidths were $0.23 \mathrm{~mm} / \mathrm{s}$, to be compared with the natural width of $0.19 \mathrm{~mm} / \mathrm{s}$. Linearity of the velocity scale was better than $0.1 \%$, and calibrations were stable to $0.2 \%$ over periods of order one week. Permanent magnets were used to supply small magnetic fields $(0.043 \mathrm{~T})$ in a direction transverse to the gamma beam. High magnetic field spectra were obtained using a splitcoil superconducting magnet with the gamma beam directed normal to the field. A spectrum typically took $10 \mathrm{~h}$ to collect. For measurements at temperatures above liquid helium temperature, the sample was placed in a small counter Dewar on a heated copper finger, with silicone grease to ensure good thermal contact. The temperature, measured with a gold iron-chromel thermocouple, has an estimated uncertainty of $3 \%$.

\section{RESULTS AND ANALYSIS}

We recorded Mössbauer spectra of compound $A$ under a variety of temperature and magnetic field conditions. The data are shown in Figs. 1, 2, and 3 along with simu- 


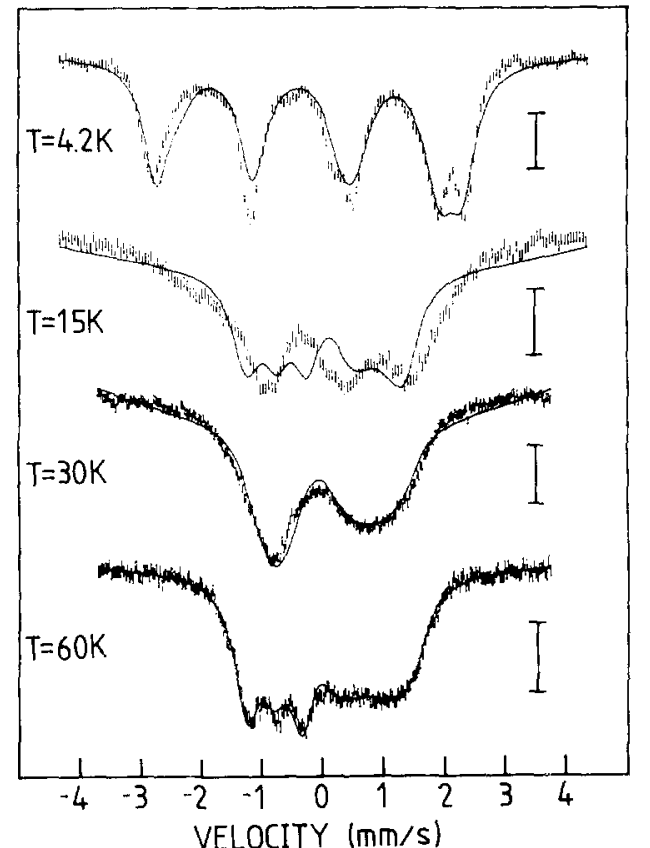

FIG. 3. Mössbauer spectra of compound $\mathrm{A}$ in a transverse magnetic field of $6 \mathrm{~T}$ at temperatures indicated. The solid lines are calculations based on the $S=3 / 2$ spin Hamiltonian of Eqs. (1) and (2). The bars at the right indicate $1 \%$ resonant absorption.

lations (solid lines) described below. The top two spectra of Fig. 1 were taken in the absence of an applied magnetic field. Both spectra show evidence for magnetic interactions. The $77 \mathrm{~K}$ zero field spectrum consists of a single asymmetric quadrupole pair. We fitted this with two Lorentzians, allowing the positions, intensities, and half-widths to vary in order to minimize the sum of the squared residuals. The fits yielded $\delta$, the isomer shift with respect to metallic iron, $0.06 \mathrm{~mm} / \mathrm{s}$ and $\Delta E_{Q}$, the quadrupole splitting, $1.62 \mathrm{~mm} / \mathrm{s}$, suggesting that the iron in compound $A$ is $F e(I V)$, as in HRP-I and compound ES (see Table I). The large linewidth $\Gamma \sim 0.50 \mathrm{~mm} / \mathrm{s}$, and the asymmetry of the $77 \mathrm{~K}$ spectrum shows that even at this temperature the electron spin relaxation rate is not fast enough to wash out the magnetic interaction. The $4.2 \mathrm{~K}$ spectrum has two components, a fast relaxing quadrupole doublet with splitting and isomer shift approximately the same as the $77 \mathrm{~K}$ spectrum, and a broad diffuse spectrum characteristic of a slowly relaxing half-integer spin system. ${ }^{17}$ The application of a small magnetic field $\left(H_{\mathrm{apo}}=0.043 \mathrm{~T}\right)$ at $4.2 \mathrm{~K}$, however, is enough to decouple the electron and nuclear spins as shown in the bottom spectrum of Fig. 1.

The Mössbauer spectra in the absence of an applied magnetic field suggest that the integer spin $\mathrm{Fe}$ (IV) is coupled to a half-integer spin. Previous magnetic susceptibility measurements ${ }^{12}$ indicate a moment of $4.2 \mu_{B}$, or slightly larger than that expected for an $S=3 / 2$ system. Thus it appears that the iron $S=1$ is tightly coupled to a radical $S=1 / 2$. In order to test this hypothesis and to quantify the magnetic interaction we fitted ${ }^{18}$ the Mössbauer spectra using a spin Hamiltonian of the form:

$$
x=\mathbf{I} \cdot \tilde{V} \cdot \mathbf{I}+\mathbf{S} \cdot \tilde{A} \cdot \mathbf{I}-g_{N} \beta_{N} \mathbf{H} \cdot \mathbf{I}+\mathbf{S} \cdot \tilde{D} \cdot \mathbf{S}+\beta \mathbf{H} \cdot \tilde{g} \cdot \mathbf{I},
$$

where the first two terms are the electric and magnetic hyperfine interactions, respectively. The third term is the nuclear Zeeman interaction and the last two terms are the electronic zero field and Zeeman interactions. The model assumes that the hyperfine interactions and the electronic zero field splitting all share the same principal axis frame and with it we calculate Mössbauer spectra for either the fast or slow electronic relaxation regime.

For applied magnetic fields above $\sim 100 \mathrm{G}$ the electrons are effectively decoupled from the nucleus. We may then diagonalize the electronic Hamiltonian [the last two terms of Eq. (1)] separately and solve for the spin expectation values of the electronic states. In the fast relaxation limit the thermal average of these spin expectation values is then substituted in the nuclear Hamiltonian [the first three terms of $\mathrm{Eq}$. (1)] and the Mössbauer spectrum is calculated. In the slow relaxation limit, a Mössbauer spectrum is calculated using the spin expectation value of each of the electronic states and an appropriate Boltzmann average of these spectra is taken. In the absence of an applied field the complete nuclear plus electronic Hamiltonian [first, second, and fourth terms of $\mathrm{Eq}$. (1)] must be diagonalized.

The iron $S=1$ spin could couple to the radical $S=1 / 2$ spin to form a total spin of either $3 / 2$ or $1 / 2$. We attempted to fit the data using the above Hamiltonian for both cases, and found $S=3 / 2$ to be clearly the better choice. Our experience with other porphyrin model compounds and a large number of simulations lead us to the conclusion that the iron in compound A occupies a site of nearly axial symmetry, thus we set the electric field gradient asymmetry parameter $\eta$ and the electronic zero field parameter $E / D$ both equal to zero. A further assumption of an isotropic $g=2$ simplifies the Hamiltonian thus

TAB I. Comparison of the $S=1$ spin Hamiltonian and Mössbauer parameters of various compounds.

\begin{tabular}{|c|c|c|c|c|c|c|}
\hline Compound & $A_{1}^{*}(\mathrm{~mm} / \mathrm{s})^{2}$ & $A_{\varepsilon}^{*}(\mathrm{~mm} / \mathrm{s})$ & $D\left(\mathrm{~cm}^{-1}\right)$ & $\Delta E_{Q}(\mathrm{~mm} / \mathrm{s})$ & ${ }^{\delta_{\mathrm{Fe}}}(\mathrm{mm} / \mathrm{s})$ & Reference \\
\hline A & 1.50 & 0.67 & 18.6 & 1.62 & 0.06 & Present work \\
\hline $\mathrm{ES}$ & 1.27 & 0.44 & 19.2 & 1.55 & 0.05 & 19,20 \\
\hline $\operatorname{HRP}(\mathrm{I})$ & 1.15 & 0.47 & 22.2 & 1.25 & 0.08 & 4 \\
\hline $\begin{array}{l}\text { Theory } \\
(\Delta=7 \zeta)\end{array}$ & 1.39 & 0.42 & 16.2 & & & 19 \\
\hline
\end{tabular}

${ }^{\mathrm{a}}$ Note that $A^{*}$ refers to coupling with ${ }^{57} \mathrm{Fe}$, and that $1.00 \mathrm{~mm} / \mathrm{s}$ corresponds to a magnetic field of $\langle S\rangle(-14.76 T)$ at the nucleus. 


$$
\begin{aligned}
\mathcal{H}= & (1 / 4) e Q V_{z z}\left\{I_{z}^{2}-(15 / 4)\right\}+\mathrm{S} \cdot \tilde{A} \cdot \mathbf{I}-g_{N} \beta_{N} \mathrm{H} \cdot \mathbf{I} \\
& +D\left\{S_{z}^{2}-(5 / 4)\right\}+g \beta \mathrm{H} \cdot \mathbf{S} .
\end{aligned}
$$

All of the spectra in Figs. 1, 2, and 3 except for the $77 \mathrm{~K}, H=0$ spectrum were fitted with this Hamiltonian using the values: $S=3 / 2, \Delta E_{Q}=+1.62 \mathrm{~mm} / \mathrm{s}, \delta=0.06$ $\mathrm{mm} / \mathrm{s}, \Gamma=0.50 \mathrm{~mm} / \mathrm{s}, A_{1}^{*}=1.00 \mathrm{~mm} / \mathrm{s}, A_{k}^{*}=0.45 \mathrm{~mm} /$ s. The asterisk $\left(^{*}\right)$ indicates that the magnetic hyperfine parameters relate to the excited nuclear state of ${ }^{57} \mathrm{Fe}$.

The simplified $S=3 / 2$ spin Hamiltonian does a remarkably good job of fitting the spectra at the temperature extremes. At $4.2 \mathrm{~K}$ in an applied magnetic field only the ground state of the $S=3 / 2$ spin quartet is populated and thus relaxation rate is irrelevant. The simulations for the 30 and $60 \mathrm{~K}$ spectra were calculated in the fast relaxation limit. The 15 and $20 \mathrm{~K}$ spectra show features of an intermediate relaxation rate. Since calculations in this regime are quite difficult we have displayed fast relaxation simulations for comparison. The solid curve plotted over the low temperature zero field spectrum of $F$ ig. 1 results from the complete diagonalization of Eq. (1), and is based on the assumption of slow electron spin relaxation. It appears to reproduce most of the observed features of the spectrum. Evidently a significant part of the sample exhibits intermediate relaxation rate, giving rise to a component similar to the $77 \mathrm{~K}$ spectrum.

\section{DISCUSSION}

We have demonstrated that compound $A$ is fairly well described as an axial spin $3 / 2$ system. If we treat this as an iron complex of unit spin strongly and isotropically coupled to a spin $1 / 2$ free radical we can determine the $S=1$ spin Hamiltonian parameters of the former and compare it with the ferryl iron of ES and HRP-I. We let $S_{1}=1, S_{2}=1 / 2$, and allow these to combine to form net spin $S=3 / 2$. It is an elementary exercise in quantum mechanics to show that the spin components are related in the following manner:

$S_{1 x}=\left(\frac{S(S+1)+S_{1}\left(S_{1}+1\right)-S_{2}\left(S_{2}+1\right)}{2 S(S+1)}\right) S_{x}=(2 / 3) S_{x}$

A similar relation holds for the $y$ and $z$ components. The magnetic hyperfine interaction is proportional to $\bar{A} \cdot \mathbf{S}$ where of course $\bar{A}$ and $\mathbf{S}$ refer to the same fictitious spin. Our measurements thus imply magnetic hyperfine coupling parameters $A_{\perp}^{*}=(3 / 2) \times 1.00=1.50 \mathrm{~mm} / \mathrm{s}$ and $A_{z}^{*}=(3 / 2) \times 0.45=0.67 \mathrm{~mm} / \mathrm{s}$, relevant to a $\mathrm{spin}=1$ system. The relation between the operators for $S_{z}^{2}$ is more complex than Eq. (3), but it can be shown that a factor of 3 connects the $D$ values. Thus the $D$ relative to an $S=1$ system is $D=(3) \times 6.3=18.6 \mathrm{~cm}^{-1}$. In order to facilitate comparison we have prepared Table I, using standard methods ${ }^{19}$ to recast the compound ES data ${ }^{20}$ in spin Hamiltonian form. We note that all of the values for compound $A$ are reasonably close to those of the protein compounds, indicating that the electronic structure of the ferryl center is similar in all cases.
The spin Hamiltonian provides a convenient parametrization of the data. It is related to the more fundamental crystal field description, ${ }^{19}$ in which the energies of the various $d$-electron orbits and the spin oribt coupling constant $\zeta$ are the basic parameters. Spin-orbit mixing of the excited orbital levels with the ground level determines the nature of the ground multiplet and thus the constants of the spin Hamiltonian. Thus the parameters for ES in the table correspond to a level scheme in which the one-electron orbitals $|x z\rangle$ and $|y z\rangle$ are at energy $\Delta$ $=6.0 \zeta$ above $|x y\rangle$. Our present results fit the crystal field model fairly well if we increase the axial field parameter to $\Delta=7.0 \xi$; the resulting calculated parameters are shown in the last line of Table I. The poor agreement with the theoretical value of $A_{\varepsilon}^{*}$ is not important in view of the relative insensitivity of our measurements to that quantity. This is understood in terms of the structure of the multiplet: for most directions of applied field the effective spin has negligible $z$ component. Thus in our measurements of a randomly oriented sample only a small fraction of the sample is sensitive to $A_{z}^{*}$. It should be noted that, unlike the present case, the $A_{z}^{*}$ values of Table I are not independent experimental results in case of the proteins. Rather, they were forced to be consistent with other parameters in a set which could be related to the crystal field treatment. We must also point out that our fitting procedure employed isotropic $g$, while the crystal field theory with $\Delta=7.0 \zeta$ implies $g=2.14,2.14,1.98$ (relative to $S=1$ ). This would have a very small effect on the statistical weight assigned to various spin directions and negligible effect on the calculated spectrum.

The simple crystal field model would imply a quadrupole splitting of about $+3 \mathrm{~mm} / \mathrm{s}$ if only the valence electrons contribute to the $\mathrm{efg}$. This is about twice that observed, and it points up the difficulty of making dependable $\Delta E_{Q}$ calculations and the probable importance of bonding levels. The disagreement with simple theory is a feature which the present work shares with the treatments of the protein complexes. It is fortunate that the magnetic properties depend only on the valence electrons so that magnetic features of Mössbauer spectra can be interpreted with more confidence.

Our data and their interpretation indicate that the basic ferryl complex of compound $A$ is similar to those of ES and HRP-I, differing only in having a somewhat stronger axial crystal field. The tight coupling with a free radical to form a spin $=3 / 2$ system is demonstrated; this feature has not been observed in any analogous protein.

\section{ACKNOWLEDGMENTS}

Support of this research at the Pennsylvania State University by the National Heart and Lung Institute (NIH HL-16860 to GL) is appreciated. Support of the research at the University of Michigan by the National Institutes of Health (GM 25923 to JTG) is also gratefully acknowledged. The National Science Foundation provided funds for the purchase of a $360 \mathrm{MHz}$ NMR spectrometer (University of Michigan). 
${ }^{1}$ W. D. Hewson and L. P. Hagar, in The Porphyrins (Academic, New York, 1979), Vol. 7, pp. 295-332.

${ }^{2}$ T. Yonetani, J. Blol. Chem. 241, 2562 (1966).

${ }^{3}$ B. M. Hoffman, J. E. Roberts, C. H. Kang, and E. Margoliash, J. Biol. Chem. 256, 6556 (1981).

${ }^{4}$ C. E. Schulz, P. W. Devaney, H. Winkler, P. G. Debrunner, N. Doan, R. Chaing, R. Rutter, and L. P. Hagar, FEBS Lett. 103,102 (1979).

5J. E. Roberts, B. M. Hoffman, R. Rutter, and L. P. Hagar, J. Biol, Chem, 256, 2118 (1981).

${ }^{6}$ G. N. LaMar, J. S. de Ropp, K. M. Smith, and K. C. Langry, J. Biol. Chem. 256, 237 (1981).

7J. Fajer, D. C. Borg, A. Forman, D. Dolphin, and R. H. Felton, J. Am. Chem. Soc. 92, 3451 (1970).

${ }^{8}$ R. E. White and M. J. Coon, Annu. Rev. Biochem. 49, 315 (1980).

${ }^{9} \mathrm{~J}$. T. Groves, Adv. Inorg. Biochem. 1, 119 (1979).

${ }^{10}$ D. -H. Chin, A. L. Balch, and G. N. LaMar, J. Am. Chem. Soc. 102, 1446 (1980).
${ }^{11}$ W. F. Simmoneaux, W. F. Scholz, C. A. Reed, and G. Lang, Biochim. Biophys. Acta 716, 1 (1982).

${ }^{12} \mathrm{~J}$. T. Groves, R. C. Haushalter, M. Nakamura, T. U. Nemo, and B. J. Evans, J. Am. Chem. Soc. 103, 2884 (1981).

${ }^{13}$ G. M. Badger, R. A. Jones, and R. L. Laslett, Aust. J. Chem. 17, 1028 (1964).

${ }^{14} \mathrm{~J}$. T. Groves, and T. E. Nemo, J. Am, Chem. Soc. (in press).

${ }^{15}$ Initial samples of ${ }^{57} \mathrm{Fe}(\mathrm{TMP}) \mathrm{Cl}$ were prepared by Dr. R. C. Haushalter, cf. Ref. 12 .

${ }^{16} \mathrm{R}$. -J. Cheng, L. Latos-Grazyuski, and A. L. Balch, Inorg. Chem. 21, 2412 (1982).

${ }^{17}$ G. Lang, Q. Rev. Biophys. 3, 1 (1970).

${ }^{18} \mathrm{G}$. Lang and B. W. Dale, Nucl. Instrum. Methods 116, 567 (1974).

${ }^{19} \mathrm{~W}$. T. Oosterhuis and G. Lang, J. Chem. Phys, 58, 4757 (1973).

${ }^{20} \mathrm{G}$. Lang, K. Spartalian, and T. Yonetani, Biochim. Biophys. Acta 451, 250 (1976). 